\title{
Pan-PIM Kinase Inhibitor NVP-LGB-321
}

National Cancer Institute

\section{Source}

National Cancer Institute. pan-PIM Kinase Inhibitor NVP-LGB-321. NCI Thesaurus. Code C128615.

An orally available, small molecule and selective ATP-competitive pan-inhibitor of proviral integ ration sites for Moloney murine leukemia virus (PIM) kinases, with potential antineoplastic activity. Upon oral administration, pan-PIM kinase inhibitor NVP-LGB-321 binds to and prevents the activation of the three PIM family kinases, PIM1, PIM2 and PIM3. This prevents the activation of PIM-mediated signaling pathways and inhibits proliferation in cells that overexpress PIMs. PIMs, constitutively active proto-oncogenic serine/threonine kinases upregulated in various types of cancers, play key roles in tumor cell proliferation and survival. 